Article

\title{
Development of a Shape Replicating Draping Unit for Continuous Layup of Unidirectional Non-Crimp Fabrics on Complex Surface Geometries
}

\author{
Berend Denkena ${ }^{1}$, Carsten Schmidt ${ }^{2} \mathbb{D}$, Simon Werner ${ }^{2, *}{ }^{\circ}$ and Dietmar Schwittay ${ }^{2}$ \\ 1 Institute of Production Engineering and Machine Tools, Leibniz Universität Hannover, An der Universität 2, \\ 30823 Garbsen, Germany; denkena@ifw.uni-hannover.de \\ 2 Institute of Production Engineering and Machine Tools, Leibniz Universität Hannover, Ottenbecker Damm 12, \\ 21684 Stade, Germany; schmidtc@ifw.uni-hannover.de (C.S.); schwittay@ifw.uni-hannover.de (D.S.) \\ * Correspondence: werner@ifw.uni-hannover.de; Tel.: +49-4141-77638-204
}

check for updates

Citation: Denkena, B.; Schmidt, C.; Werner, S.; Schwittay, D.

Development of a Shape Replicating Draping Unit for Continuous Layup of Unidirectional Non-Crimp Fabrics on Complex Surface Geometries. J. Compos. Sci. 2021, 5, 93. https:// doi.org/10.3390/jcs5040093

Academic Editor:

Francesco Tornabene

Received: 2 March 2021

Accepted: 29 March 2021

Published: 1 April 2021

Publisher's Note: MDPI stays neutral with regard to jurisdictional claims in published maps and institutional affiliations.

Copyright: (c) 2021 by the authors. Licensee MDPI, Basel, Switzerland. This article is an open access article distributed under the terms and conditions of the Creative Commons Attribution (CC BY) license (https:// creativecommons.org/licenses/by/ $4.0 /)$.

\begin{abstract}
The manufacturing of large-scale structural components is still dominated by manual labor in many sectors of the modern composite industry. Efforts are being made to establish an automated layup technology for complex structural elements. Processing dry non-crimp fiber fabrics (NCF) offers great cost opportunities and high deposition rates, compared to prepreg-based technologies like automated fiber placement (AFP). Here, the fabric architecture is considered during the draping of the plane textile on curved surfaces. In this paper, the development of a draping unit for balancing fabric tension and consolidating continuously across the layup width is presented. We introduce a geometrical process model to achieve a fabric-friendly draping of the used unidirectional NCF. The shape of the resulting draping front depends on the surface geometry, the shearing of the previously laid-up textile, and the positioning of the material feed. To consolidate the fabric at the altering draping front in an automated layup process, the shape of the continuous consolidation element can be controlled by the elongation of serial soft actuators, manipulated by parallel robot kinematics. The shape replication ability of the draping unit is promising for the implementation of a continuous, fabric-friendly draping process for complex surface geometries.
\end{abstract}

Keywords: draping; fabrics/textiles; process modeling; automation; lay-up (manual/automated); soft-robotic

\section{Introduction}

The adaption of the structural design to loading conditions can significantly improve the weight-saving efficiency by utilizing anisotropic material properties of carbon-fiberreinforced plastics (CFRP) and unconventional design concepts [1]. While simultaneously improving the load-dependent design and the production process in terms of assembly time and automation level, integral stiffening structures for aircraft fuselage with intersecting double-curved profiles (Figure 1a) are suggested. Draping form-giving foam cores with pre-impregnated carbon textiles to the pre-laid fuselage skin enables a co-curing process and greatly minimizes assembly work.

Laying technologies have been established to automate the production of laminate structures. Automated tape laying (ATL) and automated fiber placement (AFP) are the most widespread in industrial applications. While modern AFP-systems are capable of laying double-curved structures, they do not reach the output of ATL-systems [2]. The Advanced Ply Placement Process (APP) [2,3] combines the advantages of AFP and ATL. Here, pre-impregnated tapes up to $300 \mathrm{~mm}$ wide (similar to ATL) are deposited directly on multi-curved tools (similar to AFP) using two gripper robots and a compacting robot. With the shape-adaptive draping unit, tapes (up to $3.5 \mathrm{~m}$ long) are consolidated on complex surfaces with inner radii down to $50 \mathrm{~mm}$ in laying direction and $200 \mathrm{~mm}$ transverse to the 
laying direction. To increase the laying capacity, a robot end effector was developed in the Azimut project [4] for storing dry textiles up to $1270 \mathrm{~mm}$ wide. The motorized draping roll adapts to the surface by five linear units. To reduce the waviness and wrinkles in the draping result, Kaufmann et al. [5] integrated an adaptive material buffer into their laying system. The adjustable deflection roller sits between the material storage and the draping roll. It compensates for the difference in length of the two tape edges during draping double-curved surfaces. By controlling the angle of the buffer roll with the calculated length difference in the textile, $75 \%$ fewer waves are deposited. Richrath et al. are using [6] seven pre-tensioned CFRP spring elements to drape pre-assembled textile cuttings to wind turbine blade molds. Zhu et al. [7] equipped their laying technology with an active shearing module, but without a draping element. Applus Laboratories $[8,9]$ has developed a continuous forming technology with three robots for the production of complex stringers from prepreg laminate. Here, the fully assembled laminate is guided on one side over the curved shape and pressed onto the shape with an inflatable roller. To ensure that the individual layers can slide against each other, the laminate is heated on both sides via electric heating blankets.

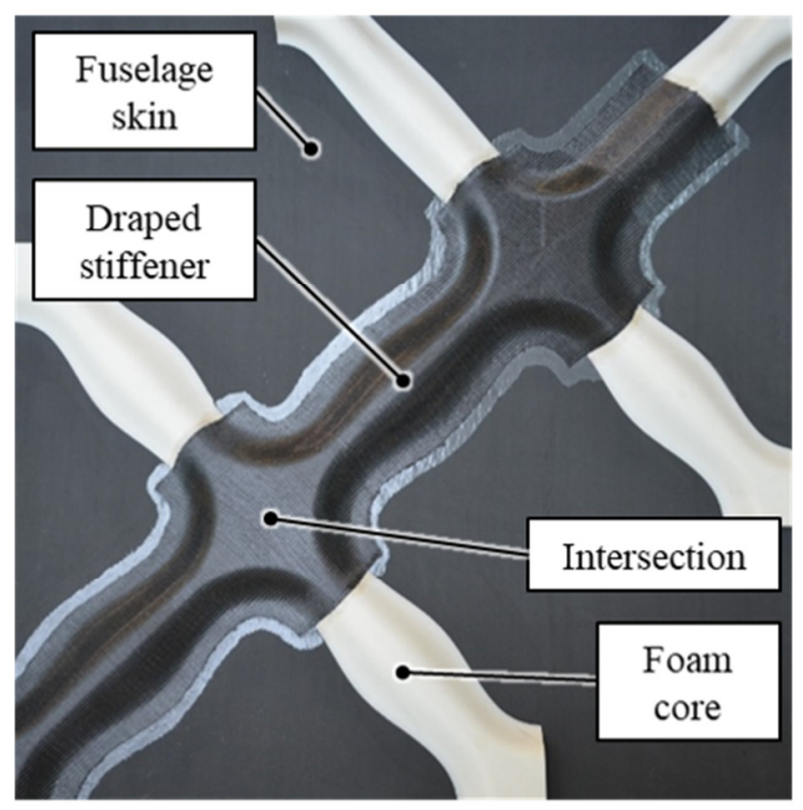

(a)

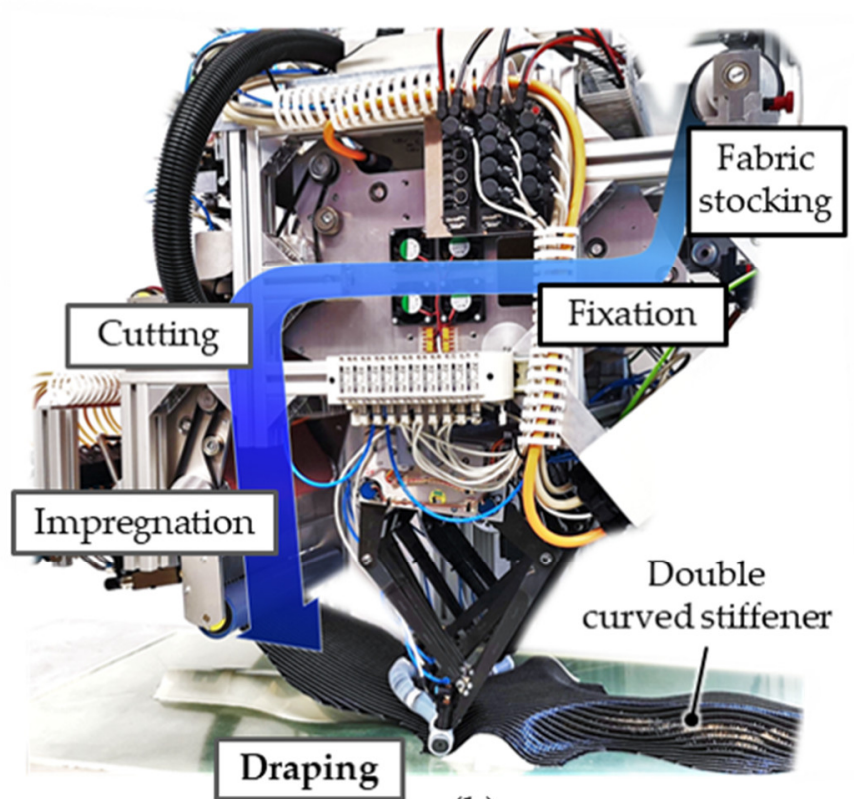

(b)

Wr/109066 @ IFW

Figure 1. (a) Unconventionally stiffened lattice composite fuselage panel; (b) robot end-effector for continuous wet draping.

While there is a range of technologies for laying up dry or pre-impregnated fabrics onto double-curved surfaces, it appears textile behavior during draping is mostly neglected in the process design. The efforts to compensate wrinkle formation $[2,5,7]$ from shearing inside the textile are insufficient for continuous draping of structures with the intended geometrical complexity. The presented approaches enable linear shearing across textile width only [5,7] or do not support a continuous material feed [2]. By using draping simulations in the process design, more textile-friendly fabric layup can be accomplished. Bruns et al. [10], therefore, use FEA-based draping simulations in the process design of thermoforming organic sheets into complex shell components. In this study, the material guidance is optimized to minimize the shear-angle in the fiber architecture and the formation of folds. Kinematic approaches of draping simulation [11] offer a fast and robust calculation of the fiber orientation without knowledge of the exact material properties. When including a limiting locking angle while shearing bidirectional textiles, predictions can be made about the drapability of complex geometries [12]. 
To automate the manufacturing of the previously mentioned double-curved stiffeners (Figure 1), a continuous wet draping process (CWD) was designed. Here, a unidirectional non-crimp fabric (UD-NCF) can be continuously fixated with thermoplastic binders, cut to contour on both sides, and impregnated with wet epoxy resin before its drapery. A robot-based layup end effector, containing functional modules for all process steps, was developed and implemented (Figure 1b). The subsequent study focuses mainly on shapeadaptive draping onto double-curved cores. Here, the research objective was to develop a draping unit for an automated continuous layup, considering the structural properties of the textile. In a kinematic draping simulation, geometric restrictions of the fiber-fabrics during forming were further investigated. Length deviations, arising when draping the textile from flat to double-curved geometry, as well as textile shearing of the pre-laid fabric, can be compensated, while consolidating at a predetermined draping front on the underlying geometry. The development of a draping unit to replicate this draping front with a nearly uniform contact surface is presented.

\section{Materials and Methods}

\subsection{Fiber Fabric Model}

The structural characteristics of a dry fiber-fabric are characterized by high pliability compared to the tensile rigidity in the fiber direction. On this basis, the fiber progression across the surface is calculated in kinematic draping simulations. In common, woven fabrics are modeled in a simplified manner as 'fishnet' with square elements [13]. Here, the fiber-length (pins) between intersections (pivoting joints) of two fiber directions stays constant, while fitting textiles to three-dimensional geometries (pin-joint-approach). The inplane deformation of the fabric becomes apparent by the shearing of the rectangular mesh cells to tilted parallelograms. Since the here used custom manufactured unidirectional non-crimp fabric from Gerster TechTex, Biberach, GER (Specifications: Table 1) gets its good drapability through a very loose weave, a new approach for kinematic draping simulation was developed and implemented. Since the polyester sewing thread is of low tension, the carbon fiber reinforcement yarn can slip through the fabric structure easily (Figure 2a). Therefore a "pin-eye"-approach is applied to the kinematic simulation of the fabric draping. By replacing the pivoting joints with eyes in the model, the carbon rovings are free to move through the fabric structure without friction, while constraining the spatial distance to each other. The shearing of the textile is subsequently defined by the shift between adjacent rovings.

Table 1. Specification of the unidirectional non-crimp fabric (UD-NCF).

\begin{tabular}{cccc}
\hline Manufacturer & Area Density & Reinforcement Yarn & Weft \\
\hline Gerster TechTex & $395 \mathrm{~g} / \mathrm{m}^{2}$ & $0^{\circ}$ CF 24k 1600 tex & Polyester: $5,4 \mathrm{weft} / \mathrm{cm}$ \\
\hline
\end{tabular}

The kinematic draping simulation (Figure 2b) is implemented in Mathworks ${ }^{\circledR}$ MATLAB. After determining the layup direction on the imported surface geometry, the fiber propagation is calculated, proceeding from the desired starting point. Therefore, geodetic lines of the surface are derived in constant distances on both sides lateral to the central layup path. Equidistant points representing the "eyes" of the kinematic approach are then computed along the geodetics. The virtual fiber paths proceed through belonging eyes. By calculating the geodetic distance between the eyes of a fiber path, the laid-up roving length is computed. 


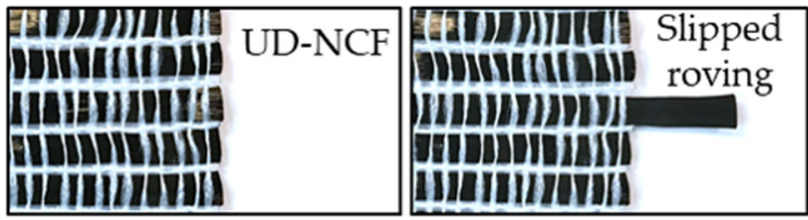

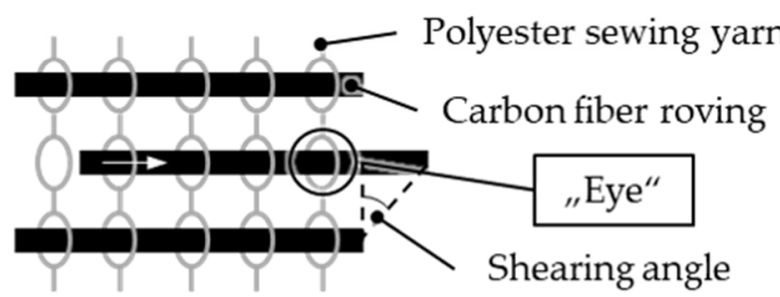

(a)

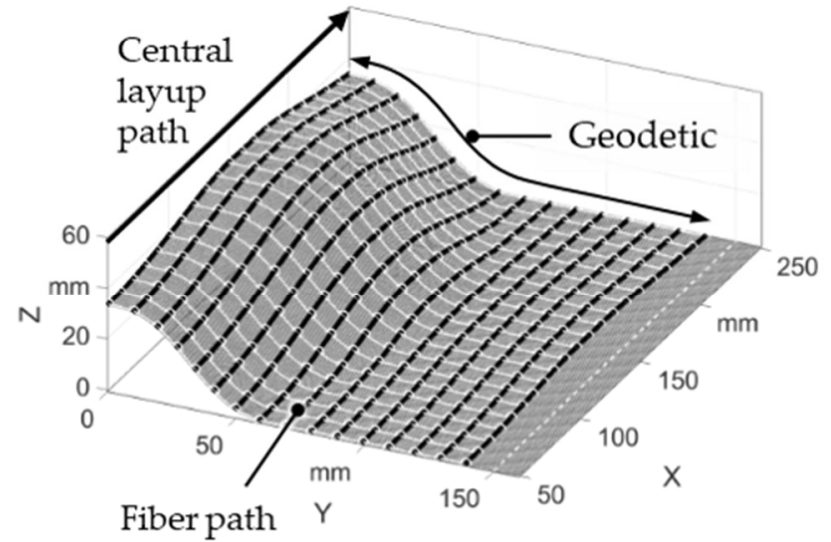

(b)
Wr/109067 C IFW

Figure 2. (a) New "pin-eye"-approach for kinematic simulation of a loose woven UD-NCF; (b) draping simulation in MATLAB.

\subsection{Experimantal Setup and Metrology}

The investigation presented in this paper is focused on a $900 \mathrm{~mm}$ long section of a double-curved stiffener with an intersection in the middle (Figure 1). The longitudinal stiffener has a varying height between $32.9 \mathrm{~mm}$ and $44.2 \mathrm{~mm}$. The rounded omega crosssection base widths range from $110 \mathrm{~mm}$ to $175 \mathrm{~mm}$. In order to minimize discrepancies in geometrical comparisons, simulation was performed on geometrical data from 3Dmeasurements of the experimental setup instead of a CAD model.

Experimental draping during the investigation was performed manually by hand layup. Spray adhesive Aerofix 2 for composite reinforcements is used to fixate the dry fabric on the mold surface.

Three-dimensional surface measurements are performed with a GOM ATOS system. The optical system captures projected blue light patterns on the surface with two cameras. $3 \mathrm{D}$ surface points are calculated from three different ray intersections with the stereo camera principle. Highly reflective surfaces (e.g., carbon fiber fabrics) are threatened with matting spray AESUB Blue to increase the quality of the 3D-image. Nominal-actual comparison of the measured geometries are performed with GOM inspect software.

\section{Results}

\subsection{Kinematic Draping Simulation}

The result of the draping simulation on the stiffener section is shown in Figure $3 b$. The textile propagation starts with $300 \mathrm{~mm}$ wide fabric at $X=0 \mathrm{~mm}$ and runs to the end of the stiffener section. The three-dimensional draping result can be unrolled to see the required outline of the fabric and the shearing pattern after draping (Figure 3a). As seen in Figure 3a, the shearing increases in layup-direction. For example, three unidirectional fiber paths are tracked to represent the undeformed fabric and across the stiffener surface. The length of the individual fiber paths deviates up to $2.25 \%$ from the maximum required fabric length $\left(\mathrm{L}_{\max }=923.7 \mathrm{~mm}\right)$. Lateral green lines represent equidistant fiber lengths across the textile surface.

To verify the kinematic model of the fabric, experimental draping tests were performed manually. To visualize the shearing of the fabric, $10 \mathrm{~mm}$ wide lateral lines are applied on the flat textile with a spacing of $50 \mathrm{~mm}$ using red spray paint. After draping the prepared textile on the molding tool by hand-layup, images of the draped fabric surface are compared with the simulation results. The simulation output lines of equidistant fiber lengths can be seen in Figures 3 and 4 as the green lines, and the resulting outline of the draped fabric is seen in Figure 4, as the blue lines. The simulation results are then compared to the surface images while keeping the perspective of the photographs in mind. As seen in Figure 4, the simulation results match the experimental draping. Deviations up to $10 \mathrm{~mm}$ in the 
equidistant fiber lengths (Figure 4a) and of roughly $14 \mathrm{~mm}$ in the outline (Figure $4 \mathrm{~b}$ ) can be attributed to inaccuracies of the hand-layup process and the spray paint application, as well as to neglecting the weft formation within the textile in the simulation approach The applied matt spray paint pattern can also be used to visualize the fiber orientation and shearing of the highly reflective carbon fibers in 3D-images (Figure 4c), optically measured with GOM ATOS. Different spray patterns, suitable 3D-image analyses, and an algorithm to quantify deviations are currently under research.
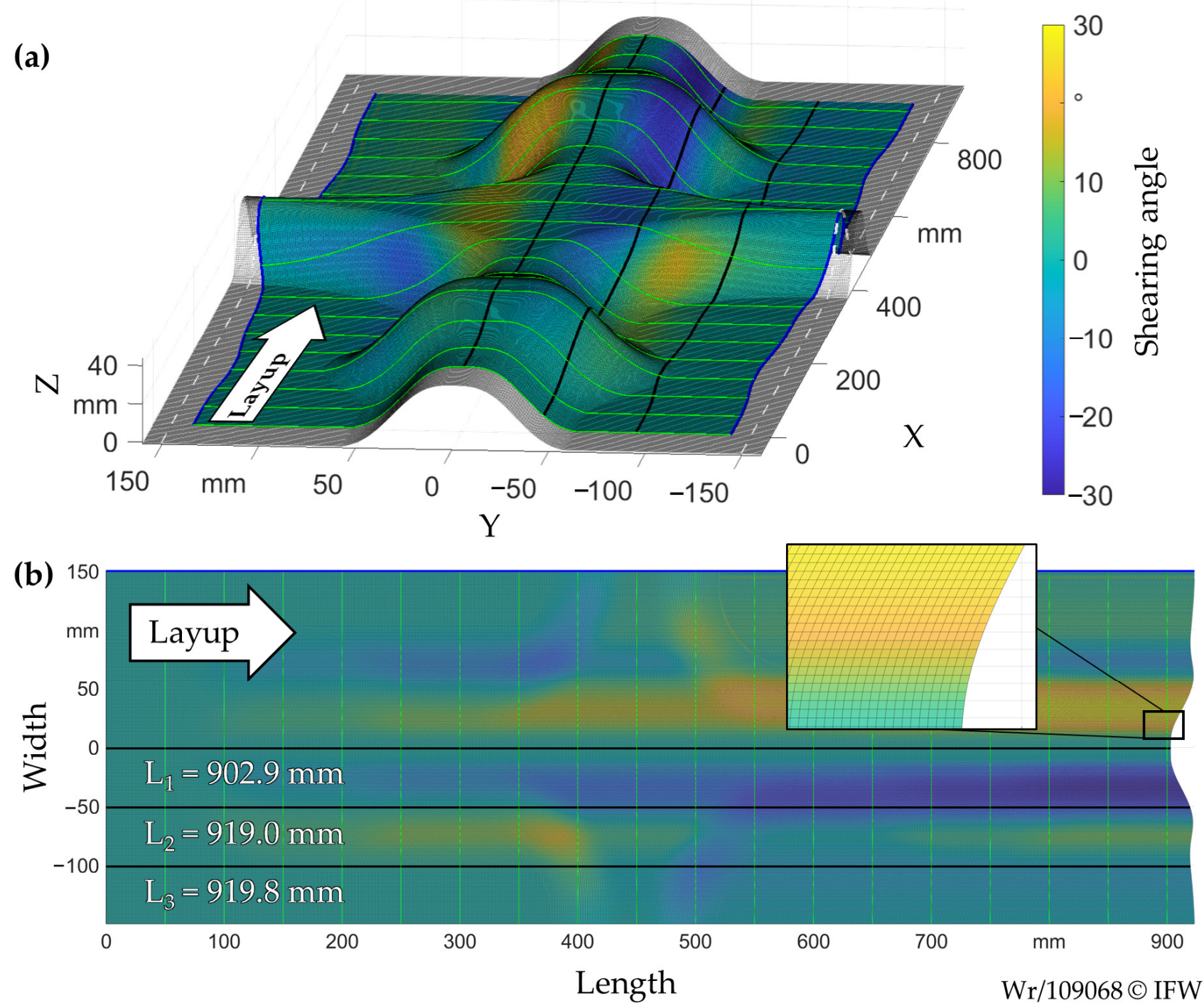

Figure 3. (a) Draping simulation on a double-curved stiffener with an intersection; (b) prediction of shearing pattern in the undeformed UD-NCF before layup.

\subsection{Process Modeling}

To achieve a good draping result, the fabric has to be kept under tension during layup. Sagging or insufficient guiding of the supplied textile can cause premature attachment to the surface and wrinkle formation. Hence, the aim of the process development for the continuous draping is to balance the textile tension throughout the width of the textile. To compensate the length deviations in the fabric directly during consolidation and to spare a material buffer (cf. [5]), a geometrical simulation of the layup process was established. 


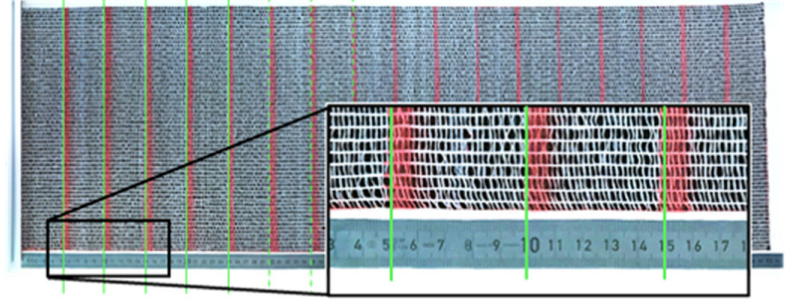

(a)

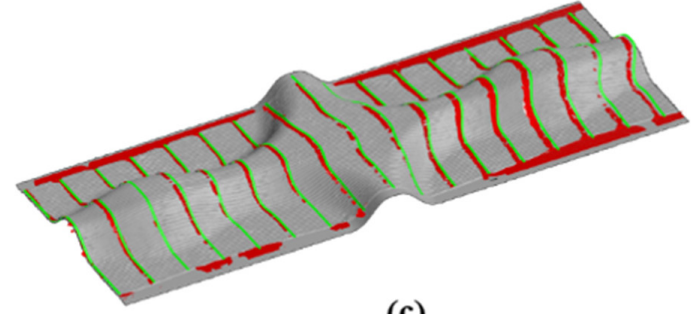

(c)

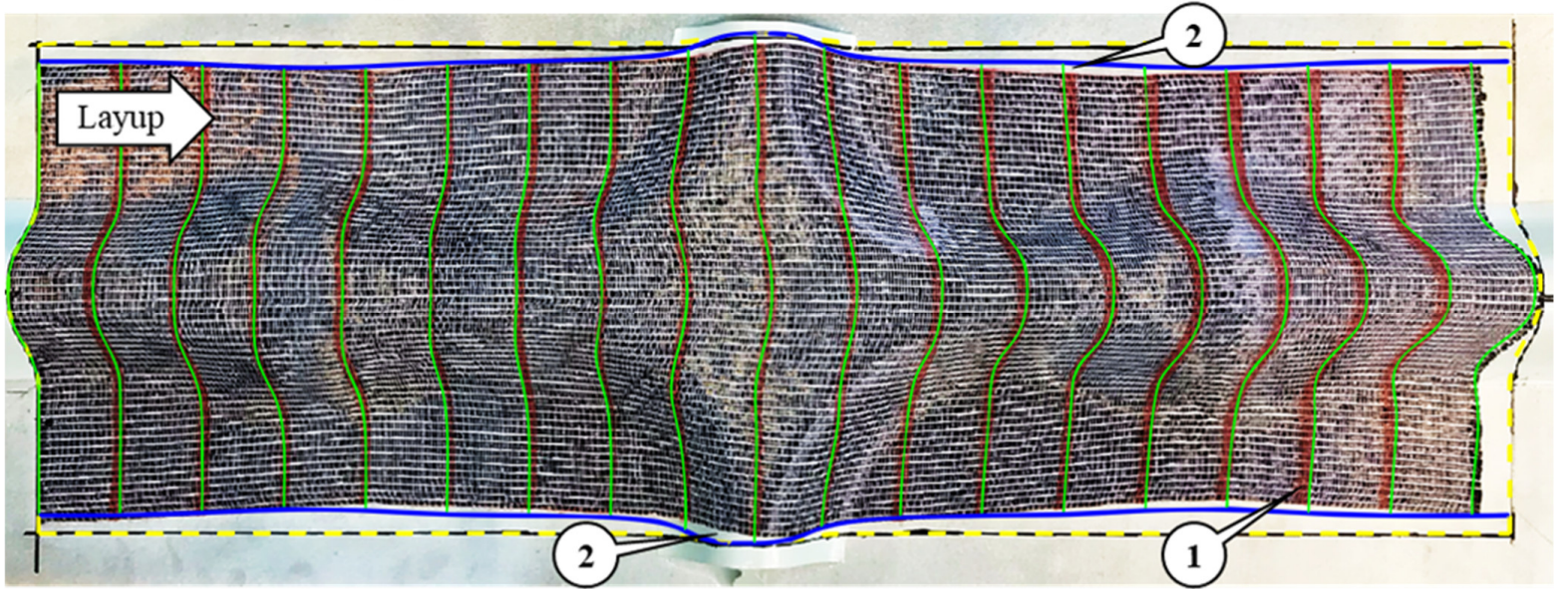

(b)

$\mathrm{Wr} / 109069$ @ IFW

Figure 4. (a) $900 \times 300 \mathrm{~mm}^{2}$ UD-NCF with lateral lines from red spray paint; (b) comparison of a 2D image of the experimental draping result with a perspective plot of the simulation; (c) comparison of the simulation with a 3D surface scan.

In order to emulate the continuous layup process, a virtual material feed is guided across the structure (Figure 5). The material feed moves in a defined path above the stiffener and ahead of the deposition area on the surface. The length deviation in the fiber paths from top undeformed material feed to the three-dimensional surface is compensated by shifting the deposition point of every fiber path in layup direction until length equilibrium is reached. A detailed description of the basis of this geometrical modeling approach is given in [14]. Finally, the accumulative length deviation of the previously laid-up fabric is considered in the calculation. Figure 5 displays the shift of the draping front to maintain an even fiber tension along the width of the fabric.

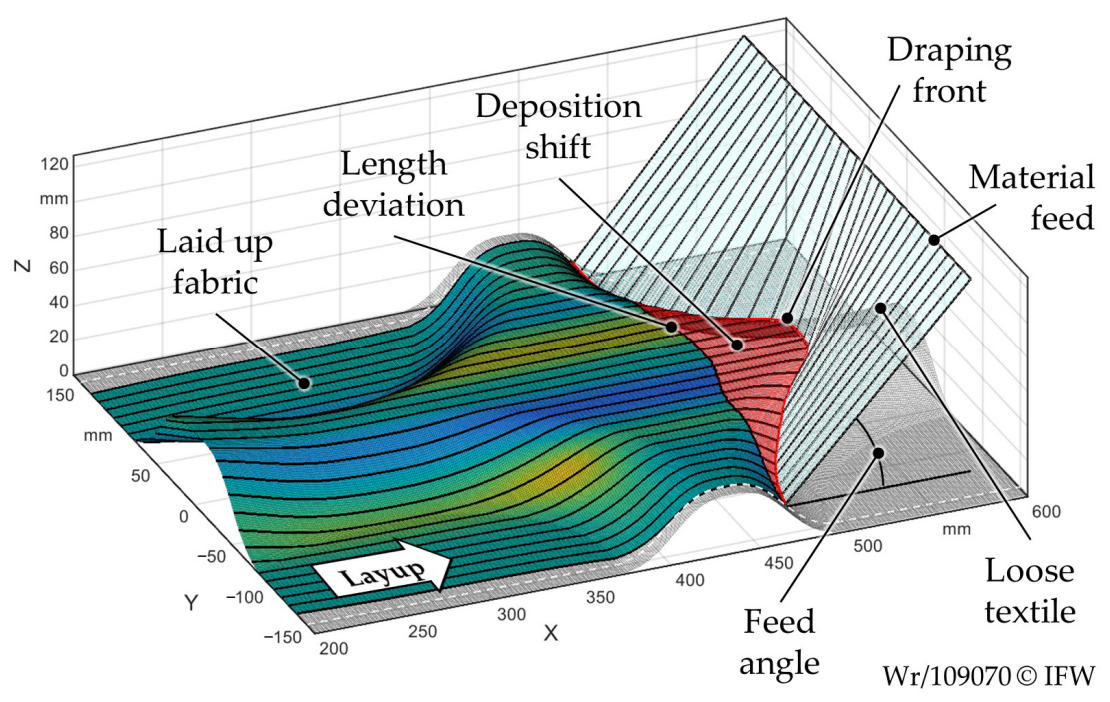

Figure 5. Deposition area on the stiffener with the shift of the draping front to maintain even fiber tension during fabric layup. 
The calculation of the draping front is performed incrementally along the structure. The shape of the draping front is constantly changing throughout the layup process. It is highly dependent on the surface geometry, but also on the relative position of the material feed and the shearing of previously laid-up fabric. Figure 6 shows exemplary results of a parameter study on the dependency of the draping front on the feeding angle and the loose textile length. The arc length of the draping front characterizes the draping situation at two different positions on the stiffener with identical geometrical characteristics. In general, the arc length of the draping front increases with shorter loose textile length and lower feeding angles. The strongest effect on the draping front characteristic throughout the layup process is the length deviation of the previously laid-up textile.
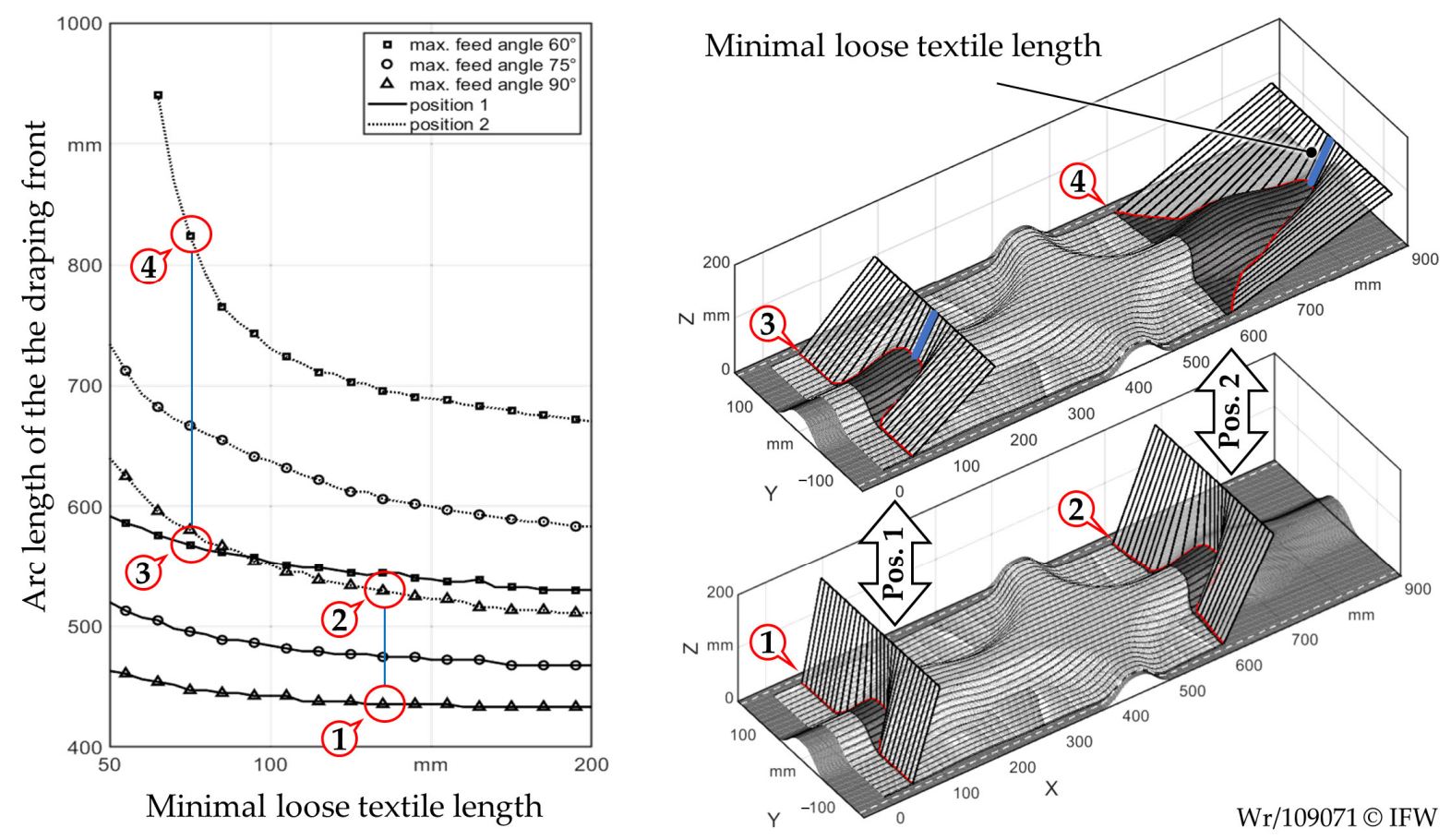

Figure 6. Dependency of the draping front on the feeding angle and the loose textile length at different positions during layup.

The geometry of the draping front is also verified in manual draping experiments. To investigate the shape of the draping front at certain draping stages, explicit conditions were derived from the simulation and reconstructed in the experimental setup to compare the 3D-measurements to the simulation results (Figure 7). To accomplish this, the undeformed textile is clamped across its width at the length of the calculated fed material. The clamping jaws are equipped with rubber-based grip tape to prevent slipping of individual roving before they left the material feed. Then the textile is draped manually to the surface of the mold. The jaws are positioned at a lower point than the actual feed position of the particular draping situation. After draping, the jaws are driven to the feed position, and the textile is ripped off the surface to a certain extent. This ensures a uniformly distributed tension across the width. Figure 7 shows the deviation between the scanned textile surface and an exported stereolithographic model from the drape front simulation at two different feed positions. The slight sagging in the loose textile is traceable to the limited adhesion of the textile to the surface. The deviation directly along the draping front of roughly $3 \mathrm{~mm}$ is caused by the bending rigidity of the fiber roving. Hence, the textile develops a radius during pull-off, while the simulation assumes a sharp angle to the surface point. The highest deviations occur in the flank region of the stiffener structure. In these regions, the highest fiber motion occurs during the rearrangement of the textile structure from its two-dimensional initial condition to the surface geometry. The geometrical model of the 
loose textile lacks the representation of the fiber drag and the internal relations lateral to the fiber direction. With increasing textile shearing during layup, the model becomes more inaccurate. Further investigations have to determine the impact of these inaccuracies on the draping result.

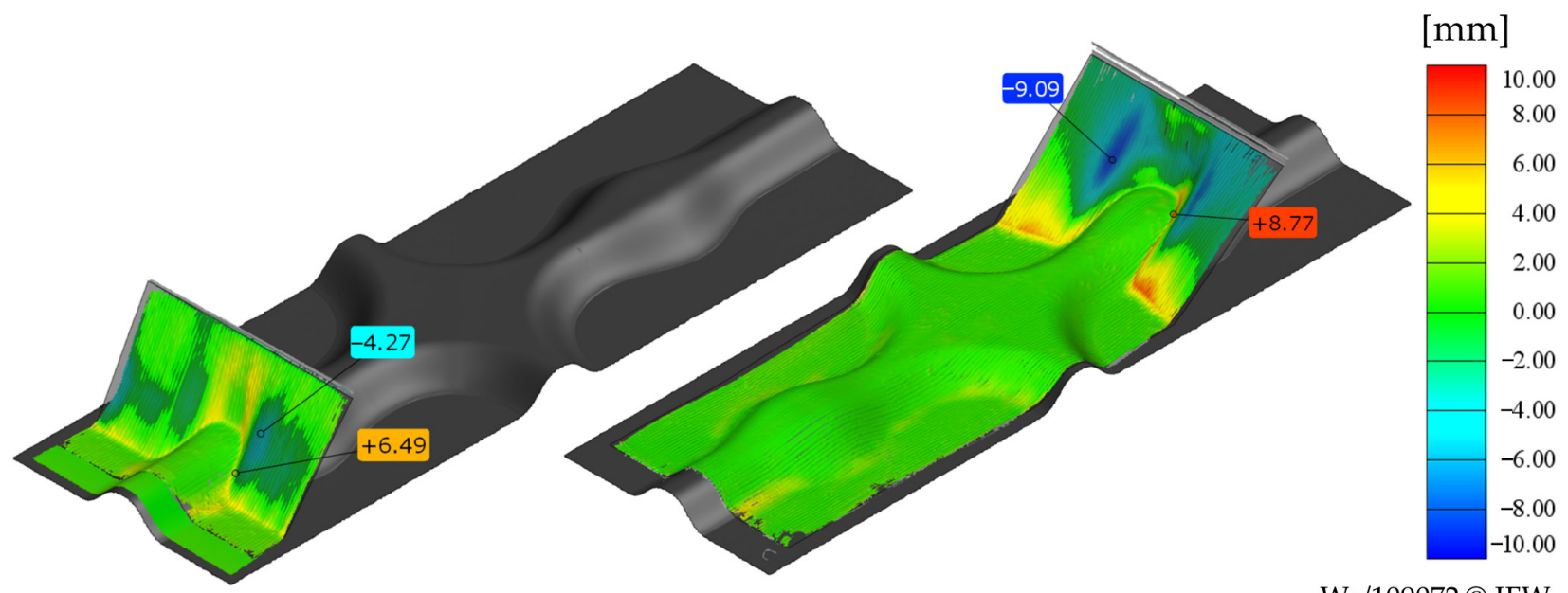

Wr/109072 @ IFW

Figure 7. Deviation analysis of draping front formation from experimental surface measurements and simulated data.

\subsection{Development of the Draping Unit}

The simulative investigation of the textile layup shows that a highly flexible draping actuator is needed to consolidate the textile at the shape-changing draping front through the process. The mechanical replication of the draping front can be solved similarly to an interpolation problem. Here, a spline function approximated the shape of the draping front best. By arranging the position of discrete support points of a pliable element, the shape of the draping front can be manipulated. To reach a smooth transition in the support points, tangential continuity has to be enabled. The altering shape of the draping front through the process requires the controllability of the arc length of the pliable element between the support points.

\subsubsection{Kinematics for Support Point Positioning}

To keep the approach for the mechanical system in the first instance simple, the positioning of the support points was discretized lateral to the layup direction. This leads to a two-axial movement of the positioning kinematic in the three-dimensional workspace (Figure 8). The aim for the construction of the kinematic was to reach a high stiffness for accurate positioning, while maximizing the workspace. Therefore, a parallel kinematic approach was chosen. With a lateral distance of $50 \mathrm{~mm}$ between each kinematic, a total of seven kinematics was realized on a layup width of $300 \mathrm{~mm}$, while still enough installation space for acceptable motorization is preserved. NEMA 17 pancake steppers with holding torque of $0.186 \mathrm{Nm}$ are connected to a synchronous belt drive with a gear ratio of 6 to move the kinematic arms. Hall effect rotary encoders are connected to the output of the belt drive to establish motion control. The arms are built as H-beams from $2 \mathrm{~mm}$ CFRP plates to ensure a lightweight, stiff structure of the kinematic. 


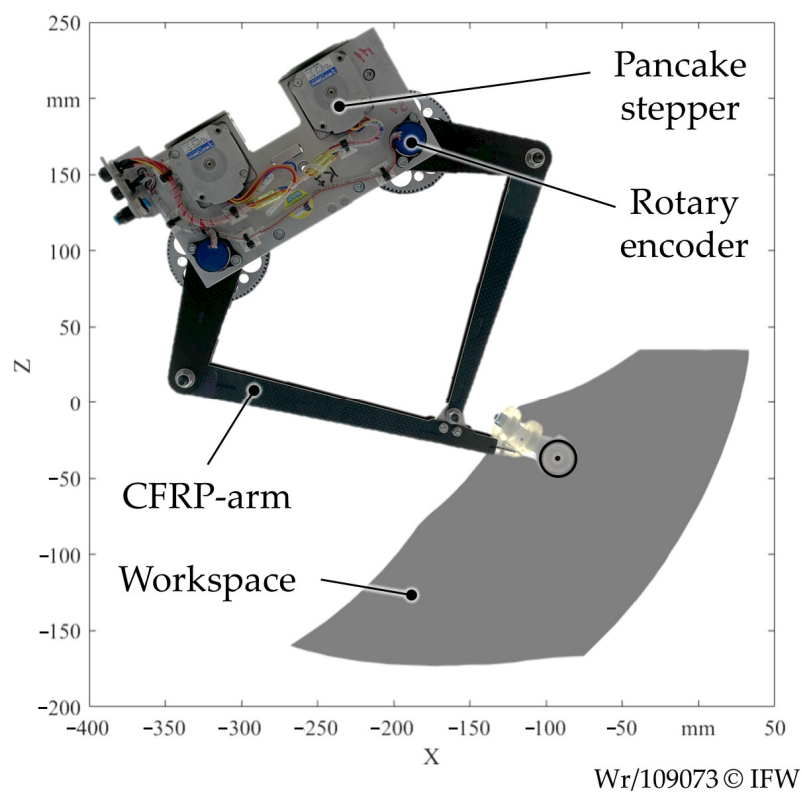

Figure 8. Parallel kinematic with two-axial workspace.

\subsubsection{Fluid-Driven Consolidation Element}

To bridge the changing distance between adjacent support points and achieve the curvature of the spline-shaped draping front, the consolidation element should offer a controllable elongation. Fluid-filled fiber-reinforced elastomeric enclosures (FREEs) are a class of soft robotic actuators and can be configured to perform the simple motion. The impermeable flexible hollow body of FREEs expands by changing the inner volume. Fiber reinforcement in the enclosure hull constrains the expansion depending on the fiber orientation [15]. A tubular elastomer body with a circular reinforcement has an unconstrained axial bendability to replicate curvatures and elongates along the axis with increasing body volume.

The field of soft robotic offers a large number of tailor-made actuators for specialized applications. Most are single-part productions with lab-scaled manufacturing processes. In order to find a reproducible manufacturing process to build robust and accurate actuators, multiple design iterations were considered. The final construction of the consolidation element consists of a continuous tubular silicon hull with integrated braided steel reinforcement and aluminum partition-elements to divide six individual chambers between the seven support points. The FREE-body is encapsulated with a flexible gliding structure made from Polytetrafluoroethylene (PTFE). Silicone was selected for the main body elastomer and PTFE for the gliding structure because of the high solvent- and temperature resistance in case of contamination during the wet draping process. Furthermore, the twocomponent room temperature curing silicone rubber Wacker Elastosil ${ }^{\circledR}$ M 4600 features very good flow properties for casting and a tensile stretch of $800 \%$ when cured. Braided steel has shown advantages over other reinforcement yarns. Especially a high resistance against abrasion prevents defects during manufacturing which causes subsequent breakage of the reinforcement in use. The compact structure of the $7 \times 7$ braiding of the Flexonit steel leader behaves like a monofilament under pressure, so no splicing occurs. The tensile load capacity is $115 \mathrm{~N}$ at a diameter of $0.36 \mathrm{~mm}$.

The production of the consolidation element (Figure 9c) consists of five manufacturing steps. The first step is to cast a silicone layer on the cylindrical core (Figure 9a) with a length of $340 \mathrm{~mm}$, an inner diameter of $21 \mathrm{~mm}$, and a wall thickness of $1 \mathrm{~mm}$ by injection molding. After the silicone is cured, the outer mold halves are opened. Before the reinforcement can be wound around the silicone, the silicone surface is sanded and treated with the silicone primer Wacker G 790 to ensure the best adhesion. Two reversed fiber coils with a pitch of $3 \mathrm{~mm}$ are wound on the silicone layer still remaining on the mold core. With this pitch 
instead of separate rings, near circular reinforcement can be achieved in an automated winding process. The core has seven radial setscrews on one side at the axial position of the partition-elements to deflect the winded yarn, yielding a reinforcement-free section. With this, the fluid feed can be attached here without the need to cut the braided steel. In the third manufacturing step, a second layer of silicon is molded around the winded inner layer to fixate and guard the reinforcement, as well as supplying a smooth outer surface. The $3 \mathrm{~mm}$ winding pitch offers enough surface area to achieve a proper attachment to the inner silicone layer. To demold the cured reinforced actuator hull, the core can be split at $2 / 3$ of the length. In preparation to install the partition walls inside the tube, the inner surface is sanded and treated with the previously mentioned primer. After the partition elements are pushed inside the tube, the segmented actuator is transferred to a fixture frame (Figure 9b) to glue the partition elements inside the elastomeric hull. Silicone glue Wacker Elastosil ${ }^{\circledR}$ E43 is injected in dedicated glue channels of every partition element through nozzles to seal the fluid chambers and creates a strong, pressure-tight connection to the positioning kinematics. The PTFE gliding structure around the actuators consists of two parts. At the stiff regions, where the partition elements are situated, a milled ring element is pushed to the support point position. The hollow chamber is wound with a PTFE-string with a $2 \mathrm{~mm}$ diameter to enable the axial movement of the actuator.

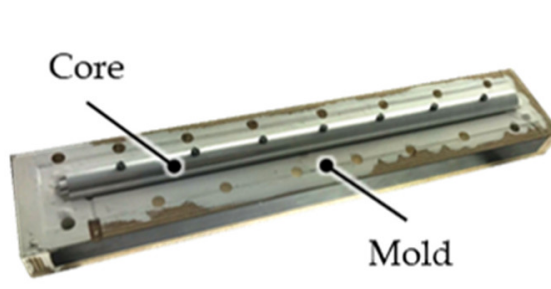

(a)

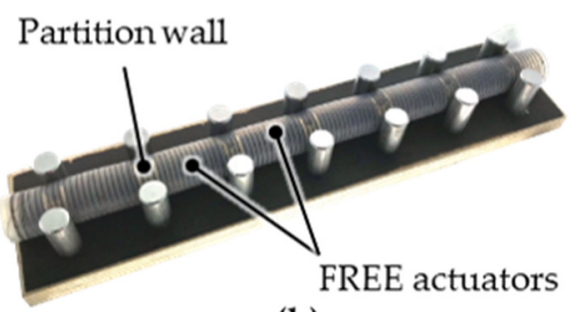

(b)

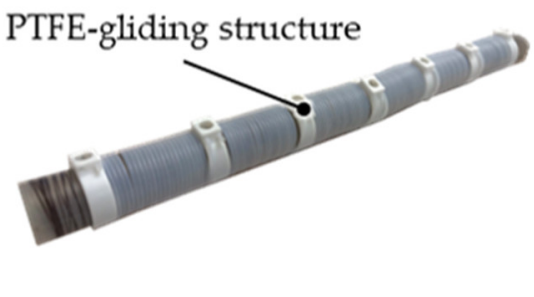

(c)

$\mathrm{Wr} / 109074 \odot \mathrm{IFW}$

Figure 9. (a) Mold half and core for inner silicone hull; (b) frame for gluing the partition walls; (c) consolidation element with assembled gliding structure.

\subsubsection{Pivoting Connection for Tangential Continuity}

The connection (Figure 10) between the consolidation elements and the parallel kinematics integrates several functionalities. Foremost, the connection has to ensure accurate positioning of the support points, while at the same time enabling a tangential continuity while replicating the spline-shape. A built-in fluid coupler directs air through a hollow shaft into the pressure chamber. The hollow shaft is pivoted to enable self-adjusting of the tangential angle at the support points. Because the precise measurement of the curvature of the consolidation element is not mechanically achievable, the measurement of the tangential angles at the known support point positions allows an approximation of the deformation. Therefore, conductive rotary potentiometers are connected to the hollow shaft.

\subsubsection{Investigation of Shape Replication Ability}

Finally, to validate the ability of the draping unit to replicate the desired draping front shape, the consolidation element in a static deformed state with the optical GOM ATOS system is measured. It is placed on the mold surface to accurately restrict the deformation as in the draping process. In Figure 11, the deviation between the measured geometry and the desired shape from the simulation is shown. Due to the restricted accessibility inside the experimental setup, only the front half of the consolidation element could be measured optically. As can be seen, the results of the replicated shape look promising. With the rotational axis in the connection elements, tangent continuity is reached between the soft actuators. The consolidation element, thus, develops a smooth curvature, as was intended 
by the interpolation approach. The highest deviations, which occur in the center of the elastomeric enclosures, can be attributed to the simplified control model. For this setup, pressurized air for actuation and a linear pressure-to-elongation-model for the soft actuator motion was used. Although efforts to characterize the motion behavior of the actuators already showed non-linear properties with a distinct hysteresis, a more accurate model was not implemented in the control system yet. In Figure 11a, the negative deviation in a concave region of the surface and the positive deviation in a convex region can be referred to as over-actuation. Here the adjusted air pressure leads to a greater elongation needed to replicate the curvature. In Figure 11b, the contrary is the case. Under-actuation leads to positive deviations in concave regions and negative deviations in convex regions. Further investigations on the motion behavior and the force distribution through the connections of the soft actuators are being undertaken.

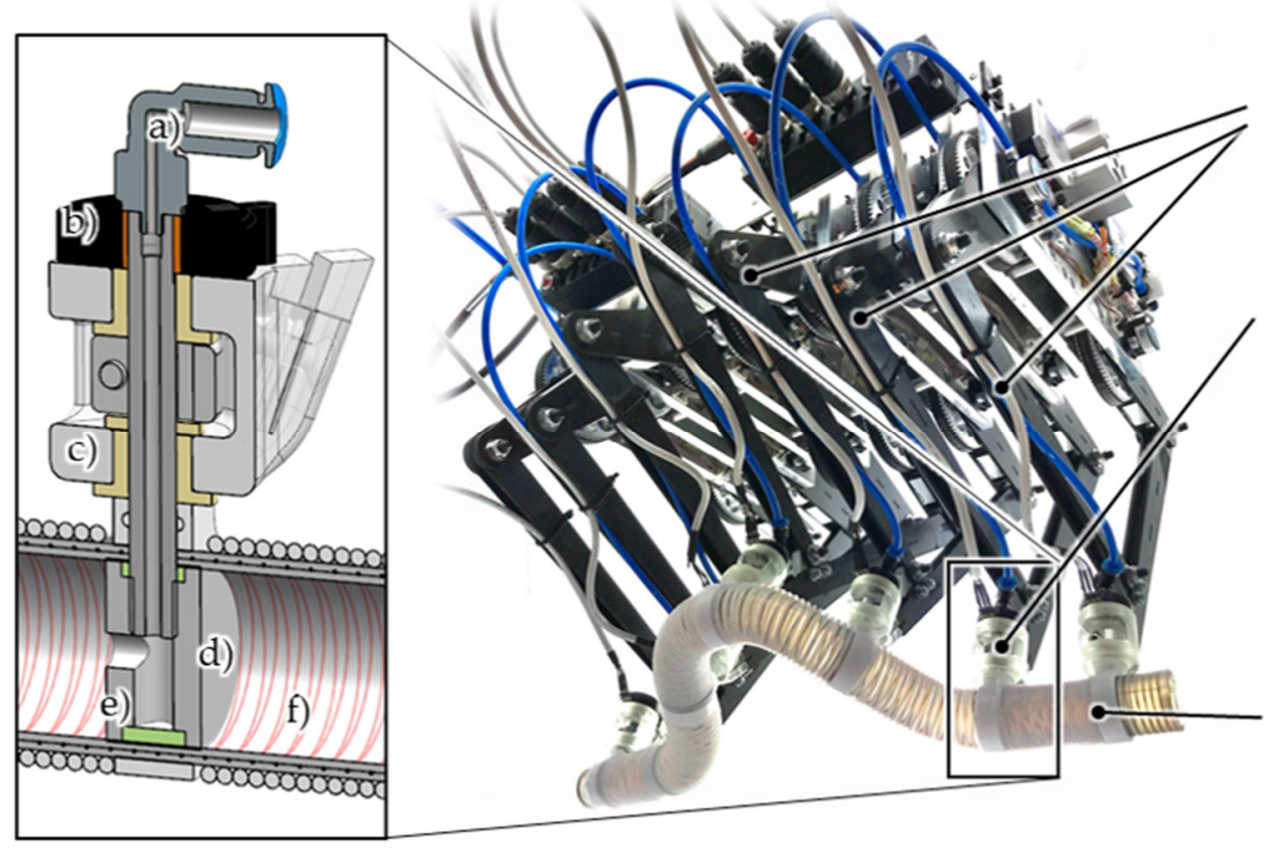

Parallel

kinematics

Integral connection

a) fluid coupling

b) potentiometer

c) bearing

d) partition wall

e) chamber sealing

f) actuator hull

\section{Consolidation}

element

Figure 10. Shape replicating draping unit with a sectional view of the connection.

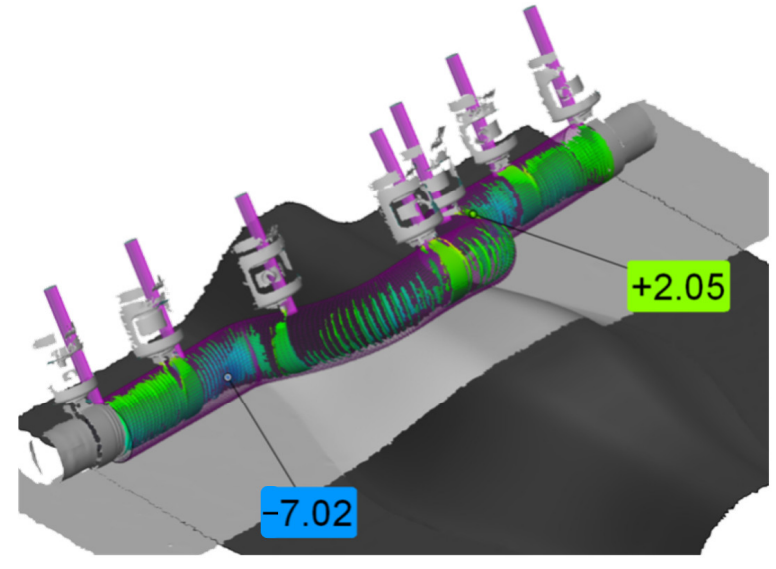

(a)

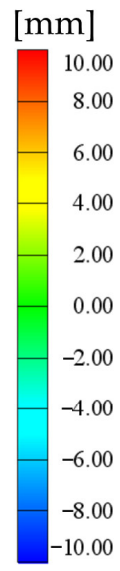

$-10.00$

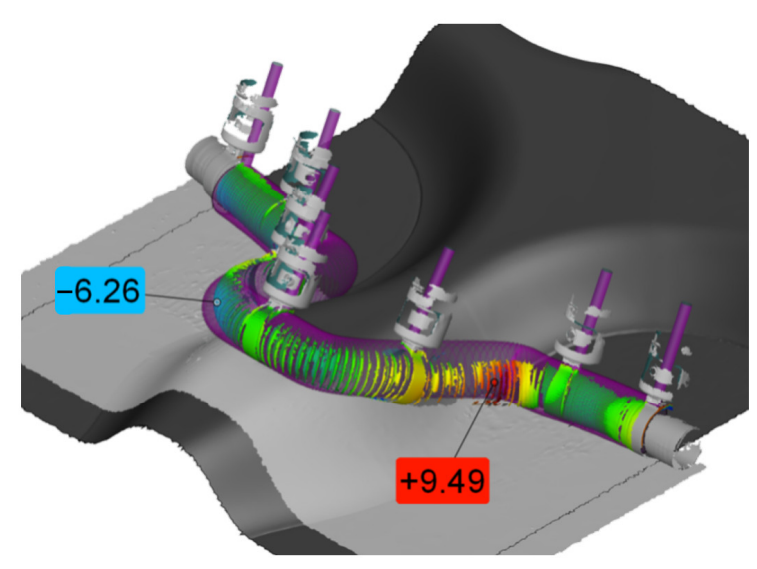

(b)
$\mathrm{Wr} / 109076 @ \mathrm{IFW}$

Figure 11. Deviation analysis of the deformed consolidation element with the simulated shape with areas of over-actuation (a) and under-actuation (b). 


\section{Discussion and Conclusions}

In this work, the simulation-based development of a shape replicating draping unit for automated layup of fiber fabrics on complex geometrical structures is presented. A previously published modeling approach to find a consolidation line to balance the fiber tension during continuous layup was extended to take the shearing of previously laid-up textile into account. To calculate the shearing, a "pin-eye"-model of the loose woven unidirectional non-crimp fabric was implemented into the kinematic draping simulation. The investigation of the experimental draping results showed high conformity between the fabric behavior and draping simulations based on the "pin-eye"-model. With increasing in-plane deformation of the fabric while draping, the neglection of friction and the associated true weft formation leads to advancing inaccuracies of the simulation. However, the geometrical approach offers high calculation speeds compared to FE-based draping simulations. This enables model-based controls of the draping front depending on fabric tension balance during layup. In addition, the complex interdependency between the material feed positioning, the surface geometry, and the draping front shape will be part of further investigation to derive path planning principles for the continuous draping process.

To replicate the calculated draping front mechanically, a flexible consolidation element with seven support points was developed. Two-axed parallel kinematics position the stiff partition walls of a six-chambered fluid-driven soft actuator to bring the consolidation element into the desired shape. Tangential continuity is reached through rotational-free connections of the kinematic arms and the elongation of actuated fluid chambers. The replication ability of the desired draping front shape was validated through deviation measurements of the deformed draping unit. The analysis showed inaccuracies in the die pressure-control model of the FREE-actuators, which lead to under- and over-actuation. Nonetheless, the overall shape replication has already reached a very good agreement with the desired shape at the draping front. Future work will concentrate on the characterization and modeling of the motion and contact force behavior of the silicone-based soft actuators to further improve the accuracy of obtaining the desired shape in a dynamic draping process.

Author Contributions: Conceptualization, C.S. and S.W.; methodology, S.W.; validation, S.W. and D.S.; formal analysis, S.W.; investigation, S.W. and D.S.; writing-original draft preparation, S.W.; writing-review and editing, B.D. and C.S.; visualization, S.W.; supervision, B.D. and C.S.; project administration, B.D. and C.S.; funding acquisition, B.D. and C.S. All authors have read and agreed to the published version of the manuscript.

Funding: The authors thankfully acknowledge the financial and organizational support of the project FlexProCFK by the federal state of Lower Saxony and the European Regional Development Fund (ERDF). The publication of this article was funded by the Open Access Fund of the Leibniz Universität Hannover.

Data Availability Statement: The data presented in this study are available on request from the corresponding author.

Conflicts of Interest: The authors declare no conflict of interest.

\section{References}

1. Deniz, O.; Horst, P.; Schmidt, C. Production-based Multi-criteria Design Optimisation of an Unconventional Composite Fuselage Side Panel by Evolutionary Strategies and a Surrogate Model of Manufacturability Analysis. In Proceedings of the 11th World Congress on Structural and Multidisciplinary Optimisation, Sydney, Australia, 7-12 June 2015.

2. Szcesny, M.; Heieck, F.; Carosella, S.; Middendorf, P.; Sehrschön, H.; Schneiderbauer, M. The advanced ply placement process-an innovative direct 3D placement technology for plies and tapes. Adv. Manuf. Polym. Compos. Sci. 2017, 3, 2-9. [CrossRef]

3. Helber, F.; Amann, A.; Carosella, S.; Middendorf, P. Intrinsic fibre heating: A novel approach for automated dry fibre placement. IOP Conf. Ser. Mater. Sci. Eng. 2018, 406, 012064. [CrossRef]

4. Niefnecker, D. AZIMUT-Automatisierung Zukunftsweisender Industrieller Methoden und Technologien für CFK-Rümpfe, Abschlussbericht; Laufzeit des Vorhabens: 01.01.2010-30-06.2014; Premium AEROTEC GmbH: Augsburg, Germany, 2014. [CrossRef]

5. Kaumfann, P.; Braun, G.; Buchheim, A.; Malecha, M. Automated Draping of Wide Textiles on Double Curved Surfaces; SCITEPRESSScience and Technology Publications: Augsburg, Germany, 2019. 
6. Richrath, M.; Franke, J.; Ohlendorf, J.-H.; Thoben, K.-D. Effector for automated direct textile placement in rotor blade production. Light. Des. Worldw. 2017, 10, 42-47. [CrossRef]

7. Zhu, S.; Magnussen, C.J.; Judd, E.L.; Frank, M.C.; Peters, F.E. Automated Composite Fabric Layup for Wind Turbine Blades. J. Manuf. Sci. Eng. 2017, 139, 061001. [CrossRef]

8. Gardiner, G. Automated Preforming, Part 2: Glide Forming. Available online: https://www.compositesworld.com/blog/post/ automated-preforming-glide-forming (accessed on 5 February 2020).

9. Redondo, C.B. A+ Glide Forming System/Automatic Stringer Manufacturing Technology. In Proceedings of the SAMPE Conference, Long Beach, CA., USA, 23-26 May 2016.

10. Bruns, C.; Micke-Camuz, M.; Bohne, F.; Raatz, A. Process design and modelling methods for automated handling and draping strategies for composite components. CIRP Ann. 2018, 67, 1-4. [CrossRef]

11. Cherouat, A.; Bourouchaki, H. Numerical Tools for Composite Woven Fabric Preforming. Adv. Mater. Sci. Eng. 2013, 2013, 1-18. [CrossRef]

12. Shuai, C. Fabric Forming Simulation and Process Optimisation for Composites. Ph.D. Thesis, University of Nottingham, Nottingham, UK, September 2016.

13. Mack, C.; Taylor, H.M. 39-The Fitting of Woven Cloth to Surfaces. J. Text. Inst. Trans. 1956, 47, T477-T488. [CrossRef]

14. Denkena, B.; Schmidt, C.; Werner, S. Continuous Draping of Double Curved Geometries. In Proceedings of the SAMPE Europe Conference, Southampton, UK, 11-13 September 2018.

15. Krishnan, G.; Bishop-Moser, J.; Kim, C.; Kota, S. Kinematics of a Generalized Class of Pneumatic Artificial Muscles. J. Mech. Robot. 2015, 7, 041014. [CrossRef] 